\title{
Disease Rarity, Carrier Status, and Gender: A Triple Disadvantage for Women with Fabry Disease
}

\author{
Andrea L. Gibas · Regan Klatt · Jack Johnson • \\ Joe T. R. Clarke · Joel Katz
}

\begin{abstract}
Fabry disease is a multi-systemic X-linked genetic disorder which has progressive and deadly consequences for those it afflicts. Fabry disease symptoms are widely recognized as a substantive burden for affected males. In comparison, female heterozygotes have traditionally been viewed as relatively symptom-free, though a debate continues about whether and how much they suffer with the disease. Previous research suggests that females with Fabry disease may be triply disadvantaged in healthcare settings owing to: (1) disease rarity, (2) devalued
\end{abstract}

A. L. Gibas · J. Katz

Department of Psychology and School of Kinesiology and Health Science, York University Toronto,

Toronto, ON, Canada

R. Klatt · J. T. R. Clarke

Division of Clinical \& Metabolic Genetics,

Hospital for Sick Children,

Toronto, ON, Canada

\section{J. Johnson}

Fabry Support \& Information Group,

Concordia, MO, USA

J. Katz

Department of Anaesthesia \& Pain Management,

Toronto General Hospital,

Toronto, ON, Canada

J. Katz ( $\Delta)$

Department of Psychology, York University,

4700 Keele Street,

BSB 232 Toronto, ON M3J 1P3, Canada

e-mail: jkatz@yorku.ca

Present address:

A. L. Gibas

Department of Psychology, Simon Fraser University,

Burnaby, BC, Canada carrier status, and (3) gender. The combined effects of these three factors suggest that female heterozygotes may suffer substantially with Fabry disease symptoms. A qualitative analysis of a brief disease-specific questionnaire provides supportive evidence. The female Fabry disease participants described experiencing significant symptoms that interfered with their lives. They also described unsatisfying experiences with healthcare professionals related to disease rarity, carrier status, and gender. The results of this study corroborate the suggestion that this triple disadvantage exists and may preclude appropriate management and treatment of females with Fabry disease. The role of healthcare professionals, including genetic counselors, in remedying this disadvantage is reviewed.

Keywords Fabry disease - Lysosomal storage disorder . Sex-linked disease · Qualitative analysis · Healthcare . Female heterozygotes

\section{Introduction}

Fabry disease is a multi-systemic $\mathrm{X}$-linked genetic disorder which has progressive and deadly consequences for those it afflicts. Symptoms of the disease vary, but can include hypohidrosis (an inability to sweat), angiokeratomas (a characteristic skin lesion typically of the trunk region of the body), cornea verticillata (a whorl-like pattern of the cornea which does not impair vision), gastrointestinal problems, and acute or chronic neuropathic pain (Fukuhara et al. 1975; Kahn 1973; Ries et al. 2003). The disease causes progressive damage to multiple body systems, including the eyes, kidneys, heart, and brain (Brady et al. 2001; Schiffmann et al. 2001). Commonly, those with Fabry disease have a foreshortened life owing to complications of 
renal failure and cardiovascular or cerebrovascular disease (Breunig et al. 2003; Desnick et al. 2003; Grabowski and Hopkin 2003).

Fabry disease symptoms are widely recognized as a substantive burden for affected males (Ries et al. 2001), but the debate persists about the extent to which females are affected by the disease. The presence of sex differences in disease severity has important implications for management and treatment since females with Fabry disease may be marginalized, dismissed, and/or ignored in current medical practice. Based on a quantitative survey of the pain associated with Fabry disease, we previously suggested that females with this disease may be triply disadvantaged in healthcare settings due to: (1) disease rarity, (2) devalued carrier status, and (3) gender (Gibas et al. 2006). The combined effects of these three factors suggest that females may suffer substantially with the symptoms of Fabry disease.

\section{Disease Rarity}

The disadvantage of disease rarity within a healthcare system is evident: few medical health professionals will encounter a person with a rare disease. As a consequence, the probability of a misdiagnosis or delayed diagnosis increases, often with harmful consequences. Fabry disease is no exception with an estimated prevalence of 1:40,000117,000 in the general population (Desnick et al. 2003; Kahn 1973). Unless a family history has been identified, Fabry disease may be misdiagnosed as a more common disease that exhibits similar symptomatology (Desnick et al. 2003). Thus, individuals suffering with Fabry disease are often not accurately diagnosed until adulthood, years after the initial onset of symptoms of the disease (Brady et al. 2001; Hopkin et al. 2003). The average age at Fabry disease diagnosis is 29 years for males (Desnick et al. 2003), years after disease pathology has significantly progressed and potentially damaged multiple bodily systems (Brady et al. 2001; Schiffmann et al. 2001). Although the onset of Fabry disease symptomatology in females may be comparable to males (Ries et al. 2003), the average delay in diagnosis is 16.3 years from the onset of symptoms for females, compared with an average of 13.7 years for males (Mehta et al. 2004).

\section{Devalued Carrier Status}

The second potential disadvantage--that of a devalued carrier status-is specific to sex-linked diseases like Fabry disease. Female heterozygotes have one mutant and one non-mutant $\mathrm{X}$ chromosome - thus establishing their carrier status. This has perpetuated the impression that females with Fabry disease are clinically affected to a lesser degree than males. Female Fabry disease patients have been described as asymptomatic (Chowdhury and Holt 2001; Doi et al. 2003) or as exhibiting intermittent and mild symptoms of the disease (Doi et al. 2003; Ro et al. 1999). However, approximately $1 \%$ of females with Fabry disease were reported to have symptoms as severe as males (Baehner et al. 2003). Neuropathic pain, a frequent sequela of Fabry disease, has been reported to be experienced by $10 \%$ of female heterozygotes, and when it occurs, it is alleged to be experienced only intermittently, or only under "certain circumstances" (Chowdhury and Holt 2001).

However, the results of empirical research are beginning to challenge the view of females as asymptomatic or marginally affected. Fabry disease has been shown to account for a large number of symptomatic female carriers (Pastores and Lien 2002). By contrast, female carriers of another X-linked lysosomal storage disease, Hunter disease, are never symptomatic unless a coincidental genetic defect, such as $\mathrm{X}$-autosome translocation, causes preferential expression of a mutated gene for Hunter disease. The nature of Fabry disease symptoms may be comparable in females and males (Ries et al. 2003), with $60-70 \%$ of heterozygote females expressing traditional symptoms of the disease (Bennett et al. 2002). In a sample of female Fabry disease patients MacDermot and colleagues (2001) reported that $30 \%$ manifested multiple serious symptoms of the disease, with $70 \%$ experiencing significant neuropathic pain, a more credible prevalence than previously reported in the literature. The normal lifespan for females is also similarly foreshortened by Fabry disease (Pastores and Lien 2002). These recent findings imply that the severity and pervasiveness of Fabry disease manifestation in females may be greater than previously thought (Baehner et al. 2003).

\section{Females in Healthcare}

The third disadvantage for female Fabry disease patients is their gender. Gender inequality is a major barrier to achieving quality healthcare (Gijsbers van Wijk et al. 1991). Although women account for the greater proportion of healthcare users (Gijsbers van Wijk et al. 1991), malederived models of pathology and treatment dominate medicine (MacDougall 2002) and bias the provision of healthcare services for females. Disparities in treatment between the sexes (i.e., the "gender gap") have been consistently identified for lung, heart, and kidney disease, which are predominantly perceived as "male diseases." Evidence also suggests that females are referred less often for diagnostic or therapeutic interventions and are treated less aggressively than men (Gijsbers van Wijk et al. 1991). Differences in treatment between males and females do not disappear when controlling for other confounding variables 
such as age, disease severity, and risk factors (Gijsbers van Wijk et al. 1991). To date there are few data that directly address the issue of the provision of healthcare services for females with Fabry disease.

Each of the above identified factors: (1) disease rarity, (2) devalued carrier status, and (3) gender, have been shown to impact the quality of healthcare provided and received by patients. The aim of the present paper is to examine verbatim reports regarding pain experiences from women with Fabry disease. The data were collected in an earlier study (Gibas et al. 2006), but not previously published.

\section{Methods}

The qualitative results reported in the present paper were derived from a pilot study of the neuropathic pain experiences of males and females formally diagnosed with Fabry disease (Gibas et al. 2006). The study received ethics approval from the Research Ethics Board at the Hospital for Sick Children and the Human Participants Review Committee at York University (Toronto, Ontario, Canada).

\section{Participants}

The participants included in the present study were female Fabry disease patients who were members of a Fabry disease support group (the Fabry Support \& Information Group or FSIG; www.fabry.org). At the time of completion of this research, the FSIG was comprised of 552 members (221 males, 230 females, and 101 sex not indicated). Of the 552 members, 454 identified themselves as Fabry disease patients, and 98 as family members or of a non-specified relationship.

\section{Measures}

The data for this study were collected through a brief disease-specific questionnaire designed to assess patient perceptions of the symptoms of Fabry disease, primarily neuropathic pain. The following two open-ended questions were included on the survey, from which the present results were derived:

(1) What aspects of your life are most affected by Fabryrelated pain?

(2) How could your doctor get a better idea of the pain/ discomfort you have?

Procedure

On April 28th and 30th, and May 6th and 9th, 2003, the questionnaire was made available electronically on the discussion page of the FSIG. In order to ensure that only Fabry disease patients completed the questionnaire, participants were required to print out the form, complete it by hand, and mail it to the FSIG. A member of the FSIG reviewed the questionnaires, stripped them of any identifying information and mailed the anonymous forms to the researchers. To generate additional Fabry disease patient responses, a copy of the questionnaire was mailed to the 552 members of the FSIG.

\section{Data Analysis}

The written responses derived from the two open-ended questions were analyzed with the help of QSR NVivo (Windows, release 2.0, Doncaster, Victoria, Australia). For the descriptive analyses, an inductive coding technique was used to analyze the data (Miles and Huberman 1994). With this technique, themes arise from the raw data, rather than being deductively coded from a pre-determined theory or methodology (Miles and Huberman 1994). A strength of qualitative methodology is the process of induction, whereby themes emerge to provide grounded theory or a hypothesis, rather than the reverse (Morse 1991).

Using the process of induction, the open-ended, written responses from the female Fabry disease survey participants were independently reviewed by two coders (AG and JK) line by line, and divided into "meaning units" (Thomas 1993), to generate emergent categories. The two analysts independently coded the same data and developed their own coding structure. The preliminary coding schemes derived individually from each analyst were compared. There was broad overlap between the two analysts' coding schemes, and slight discrepancies were resolved through discussion and consensual agreement. The result of the discussion was the formation of a coherent coding framework that attempted to be most representative of the data. Accordingly, the data were subsequently recoded to a unified coding structure.

After this first revision, the two analysts met a second time to finalize the coding framework. The two analysts concurred that the finalized coding framework was appropriate, incorporating the breadth and specificity of categories inherent in the open-ended responses.

\section{Results}

Demographic variables for the female Fabry disease participants in this study $(n=51)$ indicate that the mean age of the participants was 45.9 years old $(\mathrm{SD}=13.5$; range: 22-78 years). The mean age at diagnosis of Fabry disease was 31.1 years $(\mathrm{SD}=14.0$; range: $2-57$ years). The participants resided primarily within the United States, 
although a minority was from Canada and Europe. More detailed information regarding the Fabry disease status and quantititative pain measures of this sample is provided in a previously published article (Gibas et al. 2006). No other demographic information was collected.

\section{Emergent Themes}

From the qualitative analysis, in total, seven primary themes were derived from the two open-ended survey questions (Question 1 included four main themes and Question 2 included three main themes). Overall, five themes were considered relevant for the purposes of this research and were analyzed in further detail (see Tables 1 and 2). Additional themes that were not relevant to the current purpose were not included in the results. The data for each theme are presented below, with participant data presented in quotations, and the sample size $(n)$ is provided, where available.

Question 1: What aspects of your life are most affected by Fabry-related pain?

\section{Theme 1: Defining Fabry Disease Pain}

The comments by participants reflect three critical dimensions of neuropathic pain: intensity $(n=9)$, quality $(n=11)$, and chronicity $(n=6)$. These dimensions did not appear to be mutually exclusive, as evident by one female participant's comment:

"The pain at its worst is unbearable, relentless and excruciating. It is stabbing, throbbing, constant burning sensations that do not stop until they have run their course. It could be minutes or days. ... The intensity

Table 1 Selected Themes for Question 1: What Aspects of Your Life are most Affected by Fabry-related Pain?

\begin{tabular}{|c|c|c|}
\hline Main theme & Definition & Sub-theme $(n)$ \\
\hline $\begin{array}{l}\text { Theme 1: Defining } \\
\text { Fabry disease pain }\end{array}$ & $\begin{array}{l}\text { Comments } \\
\text { regarding critical } \\
\text { dimensions of } \\
\text { neuropathic pain }\end{array}$ & $\begin{array}{l}\text { Intensity of pain }(n=9) \\
\text { Quality of pain }(n=11) \\
\text { Chronicity of pain }(n=6)\end{array}$ \\
\hline $\begin{array}{l}\text { Theme 2: Fabry } \\
\text { disease pain } \\
\text { interference with } \\
\text { multiple life } \\
\text { domains }\end{array}$ & $\begin{array}{l}\text { Comments } \\
\text { highlight life } \\
\text { domains that } \\
\text { are impacted by } \\
\text { Fabry disease } \\
\text { Pain }\end{array}$ & $\begin{array}{l}\text { Physical/bodily functioning } \\
(n=40) \\
\text { Occupational functioning } \\
(n=11) \\
\text { Relationship functioning } \\
(n=15) \\
\text { Emotional functioning } \\
(n=28)\end{array}$ \\
\hline
\end{tabular}

$n$ refers to number of comments.
Table 2 Selected Themes for Question 2: How Could your Doctor get a Better Idea of the Pain/Discomfort You Have?

\begin{tabular}{|c|c|c|}
\hline Main theme $(n)$ & Definition & Sub-Theme $(n)$ \\
\hline $\begin{array}{l}\text { Theme 3: Level of } \\
\text { satisfaction with } \\
\text { physician assessment } \\
\text { and management of } \\
\text { Fabry disease pain }\end{array}$ & $\begin{array}{l}\text { Expressed level of } \\
\text { satisfaction with } \\
\text { physician assessment } \\
\text { and management of } \\
\text { Fabry disease pain }\end{array}$ & $\begin{array}{l}\text { Satisfaction }(n=8) \\
\text { Dissatisfaction }(n=8)\end{array}$ \\
\hline $\begin{array}{l}\text { Theme 4: } \\
\text { Misconceptions } \\
\text { regarding females } \\
\text { with Fabry disease }\end{array}$ & $\begin{array}{l}\text { Comments regarding } \\
\text { encounters with } \\
\text { healthcare } \\
\text { professionals that } \\
\text { involved } \\
\text { misconceptions of } \\
\text { female Fabry } \\
\text { disease patients }\end{array}$ & $\begin{array}{l}\text { Misconceptions } \\
\text { regarding disease } \\
\text { rarity }(n=4) \\
\text { Misconceptions } \\
\text { regarding } \\
\text { devalued carrier } \\
\text { status }(n=6) \\
\text { Misconceptions } \\
\text { regarding gender } \\
(n=2)\end{array}$ \\
\hline $\begin{array}{l}\text { Theme 5: Proposed } \\
\text { remedies }\end{array}$ & $\begin{array}{l}\text { Comments involving } \\
\text { female Fabry } \\
\text { disease patients' } \\
\text { perception of } \\
\text { needed changes } \\
\text { and remedies }\end{array}$ & $\begin{array}{l}\text { Improved medical } \\
\text { training }(n=24) \\
\text { Improved patient- } \\
\text { physician } \\
\text { communication } \\
(n=6)\end{array}$ \\
\hline
\end{tabular}

$n$ refers to number of comments

of the pain diminishes to a constant pain, but the stabbing feeling is gone. There is no way to describe how this constant pain day in, day out eventually begins to affect your overall health and attitude." (Participant 025)

Descriptions from women with Fabry disease are suggestive of the intensity of their pain experiences: "this pain was and still is excruciating. The pain is so intense that it overcomes me" (Participant 2385) and "this pain I have experienced is so deep in my bones" (Participant 8521). Female participants $(n=9)$ reported "severe," "intense," "extreme" or "excruciating" Fabry-related pain (Participants $040,025,2385$, respectively). One comment, in particular, highlights the potency of one female participants' lived pain experience:

"The episodes of pain in hands and feet are impossible to describe because the pain is so personal and out of control when it comes... The severe abdominal cramping is a sudden onset pain that is scary and something you're not sure you can survive. It actually feels like it might kill you [sic] many times I have passed out-I just hope and pray if I pass out the pain will be over when I come back ... if I come back." (Participant 040)

Participants provided vivid descriptors of the quality of pain they experience $(n=11)$. Their descriptions are indicative of the burning and tingling that is characteristic 
of neuropathic pain in general. For example, the pain "... felt as though my nerve endings were short circuiting or on fire" (Participant 2385) and "... you want to get away from the burning and tingling and you can't" (Participant 040). Other qualities of neuropathic pain reported were "It is stabbing, throbbing" (Participant 025) and "once the stabbing pain subsides, my hands and feet still burn ... but do not feel like they are flaming anymore" (Participant 2385).

Participants reported experiencing their disease-related pain as being constant or "relentless" (Participant 025; $n=$ 6). The chronicity of the neuropathic pain of Fabry disease was captured by such statements as: "never a day goes by that I don't exhibit some pain" (Participant 014) and "I am in pain in one form or another most of the time" (Participant 8993). Some of the female participants have dealt with the pain of Fabry disease for a long period of time. One 32-year-old participant (Participant 2385) noted that she has lived with "incredible pain" in her extremities since the age of two. She continued, stating "I long for the day that I no longer have pain and can do the things that I long to do... I cannot think of anything but when will it stop."

Of interest, not all respondents reported that neuropathic pain was a problem. A minority endorsed no, or minimal, Fabry disease pain experiences $(n=4)$. The responses indicated "rare" (Participant 040), "very few" (Participant 060), or "no real" (Participant 079) Fabry-related pain symptoms. Or, as Participant 5296 reported, "I don't have intense pain. However mainly bothersome symptoms [sic]."

Theme 2: Fabry Disease Pain Interference with Multiple Domains of Life

The female participants' responses highlighted the multitude of life domains that have been impacted by Fabry disease pain (e.g., "My life is most affected by the pain and fatigue in my daily work, family, recreation, and interaction with friends" Participant 057 or "All aspects of my life have changed due to Fabry related pain and other symptoms" Participant 8993). According to the participants, life was significantly affected in four major domains, including: physical/bodily functioning ( $n=40$; e.g., "Some days it is even hard to hold a pen to write" Participant 006), occupational functioning $(n=11$; e.g., "I gave up a full time job ... because of the pain, fatigue" Participant 057), relationship functioning $(n=15$; e.g., "When I get the burning in my hands and feet. I cannot function at all. And it's a hard thing when you hurt so badly you can't even hold your own child" Participant 069), and emotional functioning ( $n=28$; "The stress and frustration from day to day problems and pain is enough to put my emotions on a loop!" Participant 066).
Question 2: How could your doctor get a better idea of the pain/discomfort you have?

Theme 3: Level of Satisfaction with Physician Assessment and Management of Pain

Eight women with Fabry disease commented that they were satisfied with their care and management by physicians (e.g., "I personally feel like my physicians do understand the pain" Participant 8521). Typically, these women received healthcare at specialized treatment and research centres (e.g., the National Institutes of Health). Satisfaction was equated with patient-perceived level of physician knowledge of Fabry disease. For example, "My doc has done his best to learn all he can about our disease..." (Participant 055) and "My Dr. fortunately has a very good knowledge of Fabry's. He has read all the material provided by [the drug company] and everything on the Internet, plus all the information I and other patients have dug up for him" (Participant 011). Satisfaction was also equated with physician understanding and awareness of the disease. This was evident through statements like the following:

"Personally my physicians seem to have quite an understanding .... A physician can just do so muchhe can not take the disease away ... I am very fortunate to work with a physician ... who is very much aware of Fabry's. Also, I have a wonderful nephrologist who has done much studies [sic] on this disease. They both work extremely hard and show much concern for me." (Participant 052)

Additional comments included, "I think Fabry doctors have a good idea of the characteristic pain" (Participant 012), "My doctors are well aware of the pain I'm in..." (Participant 016) and "I personally feel like my physicians do understand the pain..." (Participant 8521).

Despite the satisfaction with healthcare expressed by some participants, other responses indicated an explicit general dissatisfaction with physicians $(n=8)$. For example, one participant stated "I was diagnosed $25+$ years ago. My experience with most doctors has been that most of them do not understand the pain/discomfort of Fabry" (Participant 057). Five participants claimed that to fully understand the disease, physicians must experience the disease pain and discomfort themselves: "The only way I know is if they themselves [physicians] experienced this excruciating pain and burning" (Participant 014), "If they [physicians] could experience Fabry-related pain for just 1 hour they would get a clear picture" (Participant 057). For the remainder, dissatisfaction was expressed through refutation that physicians (or anyone) could understand the pain of Fabry disease-"Personally, I don't think anyone could get an 
idea of the extent of the pain suffered with people who have Fabry disease" (Participant 006).

Theme 4: Misconceptions Regarding Females with Fabry Disease

Almost a quarter of the sample of the females with Fabry disease $(n=12)$ reported encounters with medical health professionals that were suggestive of misconceptions regarding (1) disease rarity (i.e., ignorance of the disease and that females may be affected), (2) devalued carrier status (i.e., the dismissal of females because of their carrier status), and (3) gender (i.e., disbelief of female pain and other Fabry disease symptoms).

\section{Misconception \#1: Disease Rarity}

The rarity of the disease in the general population may contribute to the misconception that women with Fabry disease may be adversely affected by symptoms of the disease. Responses of the participants in this study suggest that this misconception has continued to prevail, despite increasing recognition in the literature of the prevalence of women experiencing Fabry disease symptoms. For example, one participant commented that “... [Physicians need] a better understanding of the disease and the fact that women are affected" (Participant 028). Another participant felt that the onus was on her to educate her physician about the disease in order to receive appropriate care: "I need to better describe my pain to her [her physician], and give her more information regarding the impact of Fabry disease on women" (Participant 041).

\section{Misconception \#2: Devalued Carrier Status}

The traditional misconception that women heterozygous for Fabry disease are asymptomatic or minimally symptomatic for the disease was evident in the participants' reports. For example, one participant commented "... the 'carrier' label has been an extreme injustice to Female Fabbers" (Participant 065). Another commented,

"It's also a problem to know I have these major complaints and discomforts and most people still stand by the fact that women don't have Fabry-it's upsetting!" (Participant 066)

Study participants wanted physicians to recognize the presence and extent of their disease-related symptoms:

"Accept the fact that even though I am a carrier and do not have obvious kidney problems, I do have other aspects of the disease that are very debilitating. Like the severe abdominal cramping and diarrhea episodes that are particularly associated with overheating." (Participant 040)

One participant acknowledged that not all healthcare professionals adhere to the 'carrier' status misconception:

"Even though I was diagnosed with Fabry [disease], I have been told over and over that I am a carrier and am asymptomatic and the medical problems are not from any particular disease. There are some that listen." (Participant 057)

\section{Misconception \#3: Gender}

Often when an examination reveals no objective evidence of disease, females are more likely than males to be regarded by physicians as less credible (Malterud 2000). The symptoms may be ascribed a psychosomatic origin. As was evident in this study, previous diagnoses given to female Fabry disease patients included psychiatric disorders; in fact, one patient reported that "they [healthcare professionals] think we're crazy" (Participant 069). Further corroborating this view, another study participant commented, "over the years I have been called a hypochondriac, a psychotic woman, and many other titles. Even now, because the doctors in my area know little or nothing of these problems" (Participant 014). A third participant stated, "Fabry's [sic] is particularly 'misunderstood' and mistaken for hypochondria due to the fact that much of what causes us to be sick can't be seen or observed" (Participant 004).

If the symptom presentation by females is not entirely dismissed by healthcare professionals, it is often considered to be less significant than similar symptom presentations by their male counterparts, as exemplified in the following passage:

"His [her physician's] very first comment after the Fabry diagnosis was "Well, you are lucky ... you're not a man...you'd have problems...women don't have any problems!" He also did not even know how to pronounce it! Now he just avoids me and placates me and leaves it up to other doctors and NIH!" (Participant 065)

The diagnosis of a male relative with Fabry disease was found to provide sufficient evidence for a diagnosis of the disorder in females-despite long-standing suffering and medical complaints by the affected females. For example, one participant commented "... many days I am so depressed because for years the doctor told me that it was all in my head. Once my son was diagnosed life has gotten a little better" (Participant 051). 
Theme 5: Proposed Remedies

Female Fabry disease participants reported that few physicians were available to appropriately treat the disease: "When I find a doctor in this area who knows more about Fabry's than I do, I will definitely be impressed and claim that doctor as my own. However, at this point, I'm still looking" (Participant 060). One female participant recounted an unprofessional encounter with her physician when she asked about his medical knowledge of Fabry disease:

"I once asked a doctor what he knew about Fabry's disease. His response to me was 'Nothing. I guess I must have skipped class that day.' (Needless to say, I never saw that Dr. again!)." (Participant 060)

Thus, overall, the majority of female participants $(n=24)$ proposed a need for improved medical education and/or training of medical health professionals regarding Fabry disease. Examples of such comments from participants include: "medical schools need to be aware of this condition" (Participant 006), "... [doctor's should] try to learn more about Fabry's and the ugly things this disease causes and makes us feel" (Participant 027), and "the doctor's [sic] need more information on Fabry's. Most physicians say they have never heard of the disease" (Participant 043).

Additionally, female participants $(n=6)$ acknowledged that at least part of the responsibility for the patientphysician relationship involved improved communication on their part. For example, one participant wrote "I need to better describe my pain to her" (Participant 041) and another, "Verbally explain, to the best of my ability, the pain that I'm experiencing" (Participant 045). An information sheet or a daily pain journal were patient-initiated methods suggested to facilitate physician understanding of Fabry-related pain.

\section{Discussion}

Fabry disease pain is notably distinctive (Scott et al. 1999), and purportedly defies description: "The episodes of pain in hands and feet are impossible to describe because the pain is so personal and out of control when it comes" (Participant 040). However, in examining the responses to a survey on neuropathic pain symptoms of Fabry disease as part of a previous study (Gibas et al. 2006), the repetition of content by many of the female Fabry disease participants suggests some agreement and consistency of experience; thus, this permits a tentative qualitative description of Fabry disease pain. The descriptions of pain symptoms provided by the study participants corroborate previous research reporting that females heterozygous for Fabry disease experience significant pain (MacDermot et al. 2001; Wang et al. 2007).

Moreover, the pain of Fabry disease influences an afflicted individuals' capacity to perform what are typically perceived as normal, everyday physical tasks (e.g., handwriting, holding hands with a partner). The comments by the female participants suggest that Fabry disease impairs social, occupational, and other important areas of functioning. This is consistent with more recent research (Wang et al. 2007), which suggests that quality of living is hampered in females with Fabry disease.

The personal descriptions of significant pain experiences combined with an apparent limited understanding on the part of physicians provides evidence that females with Fabry disease present a special problem to the healthcare profession. Upon closer examination of the verbatim reports, the results of the present study indicate that some females with Fabry disease had experiences of being ignored, dismissed, and disbelieved by healthcare professionals. Sufficient concern, and now preliminary evidence, has been adduced to support the suggestion that females with Fabry disease may be triply disadvantaged in current healthcare systems. The combination of (1) disease rarity, (2) devalued carrier status, and (3) gender appears to have contributed to this unfortunate disadvantage. Dated misconceptions of Fabry disease symptomatology and its effect on women persist. It is important to note that any impediment - whether intentional or not- to the diagnostic process will delay vital treatment and management of Fabry disease. Given the progressive nature of this disease, these misconceptions may contribute to the foreshortening of life for females with Fabry disease.

The current healthcare system may have served as a conduit for these misconceptions, thereby facilitating this barrier to diagnosis and treatment. In her seminal work on female patients with medically unexplained illnesses (e.g., fibromyalgia), Malterud (2000) described five common 'traps' healthcare professionals fall into that perpetuate delivery of suboptimal medical care. Of the five traps, three overlap with the experiences reported by the females in this study suffering from Fabry disease: the universalistic trap (i.e., considering only a single cause of symptoms for a group of patients), the psychosocial trap (i.e., ascribing symptoms that defy explanation to mental illness), and the gender trap (i.e., harboring different expectations for males and females). These traps help to explain how female Fabry disease patients may be triply disadvantaged in healthcare settings, resulting in the potential dismissal of their disease symptoms. In general, the traditional view within the medical community of females as simply disease carriers may have contributed to the dismissal of these patients' symptoms. 
First, the dismissal of Fabry disease symptoms by healthcare professionals may be likened to Malterud's (2000) universalistic trap. This occurs when a single condition is incorrectly assigned to all patients with similar characteristics; in this case, all females heterozygous for Fabry disease are seen as being asymptomatic, despite research and clinical evidence to the contrary. As Malterud suggests,

"The physician who holds a single favorite understanding and solution of unexplainedness bears the risk of objectifying a diverse group of suffering persons by turning them into a supposedly uniform crowd of "these women."' (p. 608)

One consequence of the universalistic trap is that female patients may be required to continually contest the reality of their disease experience (Eccleston et al. 1997). As indicated by Participant 065 , simply “...the 'carrier' label has been an extreme injustice to Female Fabbers." Trust between patient and physician, a necessary element in a medical setting, becomes compromised (Diamond and Grauer 1986).

As was evident in this study, previous diagnoses given to female Fabry disease patients included psychiatric disorders, likely because they were seen to exhibit "medically unexplained" symptoms (Malterud 2000). When faced with the subjective and unexplainable symptoms of Fabry disease, the physician may fall into the psychosocial trap and apply a default diagnosis of a psychiatric disorder (Malterud 2000). The comment from Participant 069 aptly highlighted this trap_- "they [healthcare professionals] think we're crazy." Indeterminate symptom presentation is more often interpreted as psychosocially-based when the patient is female, rather than male (Malterud 2000).

Malterud (2000) further describes a gender trap, which is obviously applicable to this study, exemplified by a female with Fabry disease being told by a healthcare professional that she was "lucky" that she was not a man diagnosed with Fabry disease ("Well, you are lucky ... you're not a man... you'd have problems...women don't have any problems!" Participant 065). In a sample of female chronic disease sufferers, it has been noted that,

"Even today, women's health (or illness) experiences appear full of contradictions...; they described how doctors questioned the very reality of their symptoms and sometimes misdiagnosed them because symptoms were interpreted by medical practitioners as not being serious." (Kralik et al. 2001, p. 600)

Women predominantly comprise the so-called 'heartsink patients' (Malterud 2000), or problem patients that are bothersome to physicians. Often, these patients may be dismissed as attention-seeking (Singh et al. 1981). For women with Fabry disease encountering healthcare profes- sionals, the gender trap appears to be prominent. The comments provided by the female participants in this research provide evidence that a gender bias appears to have influenced the approach of some physicians to women with Fabry disease. Gender bias in the medical profession affects subsequent diagnosis and treatment (Malterud 2001).

Of course, despite the presence of these identified traps, it would be naive to assign sole blame to the healthcare profession for the triple disadvantage of female Fabry disease patients. However, the recognition and avoidance of these traps in medical practice would help to improve care of women with Fabry disease, by impacting physicians' "treatment protocols and their prescription pads" (MacDougall 2002, p. 848). In addition, genetic counselors can assist physicians in the recognition of at-risk females when a familial male is affected, based on pedigree. It may also be important for genetic counselors-who are aware that women with Fabry disease are often symptomatic-assist their diagnosed patients in educating their own primary care providers about the disease. Genetic counselors, particularly those that specialize in metabolic clinics, could also advocate within healthcare on behalf of women with Fabry disease (e.g., provide educational seminars to other healthcare professionals). The facilitation of referrals of female Fabry disease patients to pain management specialists and other complementary therapists (e.g., acupuncturists) who are knowledgeable about the disease may also help ease debilitating pain symptoms. Lastly, genetic counselors routinely work with individuals diagnosed with a rare disease, like Fabry disease, and as such can provide appropriate psychosocial support and resources to assist those patients. With these combined efforts, a decline in the marginalization of women with Fabry disease within the current healthcare system may be realized.

It must be noted that some women in the present sample were satisfied with their treatment and had physicians who were knowledgeable and kept up to date with developments in Fabry disease research. These women primarily received care at specialized treatment and research centres (e.g., National Institutes of Health) where misconceptions and ignorance are typically less prevalent. But, for the majority of females with Fabry disease, the misconceptions have an obvious detrimental impact when seeking effective treatment in non-specialized medical settings.

\section{Study Limitations}

There are some limitations of this study. First, the results were derived from two broad questions about pain on a survey primarily targeting neuropathic pain and other symptomatology of Fabry disease. Since the survey questions did not directly or explicitly ask participants to provide experiences related to a triple disadvantage in 
the healthcare, the results may not capture the participants' experiences fully. In addition, the results were derived from a written survey, in which contextual information is limited. Personal interviews with the target sample (i.e., personal interviews or focus groups with women with Fabry disease) may provide a greater breadth and depth of knowledge regarding the issue at hand. Lastly, the sample size from which the comments were derived was small and from one source (a Fabry disease support group). Thus, the extent to which the results may be generalizable to the population of women suffering from Fabry disease is unknown.

Nevertheless, it is important to note that almost onequarter of the sample of female Fabry disease patients spontaneously described adverse encounters with healthcare professionals on questions unrelated to this issue. This suggests that a significant problem exists. The content and consistency in responses also strongly suggests that these women were overlooked, dismissed, and/or ignored when seeking medical treatment. It is possible that the one-quarter prevalence found in this study is an underestimate of the triple disadvantage experienced by female Fabry disease patients. Research that directly targets this issue, by explicitly asking women about their healthcare experiences, may provide a more reliable estimate of the prevalence of this issue. Further research in this area is needed.

\section{Conclusions}

Taken together, the results of the present study indicate that the majority of this sample of women is living with significant, life-altering neuropathy. In addition, women with Fabry disease may be triply disadvantaged in healthcare settings, owing to their (1) devalued carrier status, (2) gender, and (3) disease rarity. To remedy this triple disadvantage, genetic counselors can play a critical role in increasing awareness of this triple disadvantage in healthcare settings. In addition, it is essential for healthcare professionals to examine the delivery of their care continually and to ensure that commonplace 'traps' are not entrenched in their practice. Rather than being dismissive, it is important for healthcare professionals to conduct a closer examination of women presenting with an assortment of ambiguous symptoms. For females with undiagnosed Fabry disease, this thorough examination may result in medical treatment and management that is critical in offsetting the debilitating progression of the disease.

Acknowledgements Funds to conduct this research were provided in part by a grant from Transkaryotic Therapies, Inc. awarded to Dr. Joe T. R. Clarke. Andrea Gibas was supported in part by a
Fellowship from the Canadian Institutes of Health Research (CIHR) Strategic Training Program Grant, Pain: Molecules to Community and a CIHR Canada Graduate Scholarships Master's Award. Joel Katz is supported by a Canada Research Chair in Health Psychology at York University.

\section{References}

Baehner, F., Kampmann, C., Whybra, C., Miebach, E., Wiethoff, C. M., \& Beck, M. (2003). Baehner, F., Kampmann, C., Whybra, C., Miebach, E., Wiethoff, C.M., \& Beck, M. (2003). Enzyme replacement therapy in heterozygous females with Fabry disease: results of a phase IIIB study. Journal of Inherited Metabolic Disease, 26, 617627. doi:10.1023/B:BOLI.0000005658.14563.77.

Bennett, R. L., Hart, K. A., O'Rourke, E., Barranger, J. A., Johnson, J., MacDermot, K. D., et al. (2002). Fabry disease in genetic counseling practice: recommendations of the National Society of Genetic Counselors. Journal of Genetic Counseling, 11, 121146. doi:10.1023/A:1014545521753.

Brady, R. O., Grabowski, G. A., \& Thadhani, R. (2001). Fabry disease: review and new perspective. SynerMed Communications, 1, 1-8.

Breunig, F., Weidemann, F., Beer, M., Eggert, A., Krane, V., Spindler, M., et al. (2003). Fabry disease: diagnosis and treatment. Kidney International. Supplement, 84, S181-S185. doi:10.1046/j.15231755.63.s84.5.x.

Chowdhury, M. M., \& Holt, P. J. (2001). Pain in Anderson-Fabry's disease. Lancet, 357, 887. doi:10.1016/S0140-6736(05)71823-7.

Desnick, R. J., Brady, R., Barranger, J., Collins, A. J., Germain, D. P., Goldman, M., et al. (2003). Fabry disease, an under-recognized multisystemic disorder: Expert recommendations for diagnosis, management, and enzyme replacement therapy. Annals of Internal Medicine, 138, 338-346.

Diamond, E. L., \& Grauer, K. (1986). The physician's reactions to patients with chronic pain. American Family Physician, 34, 117-122.

Doi, Y., Toda, G., \& Yano, K. (2003). Sisters with atypical Fabry's disease with complete atrioventricular block. Heart (British Cardiac Society), 89, e2. doi:10.1136/heart.89.1.e2.

Eccleston, C., Williams, A. C., \& Rogers, W. S. (1997). Patients' and professionals' understandings of the causes of chronic pain: blame, responsibility and identity protection. Social Science \& Medicine, 45, 699-709. doi:10.1016/S0277-9536(96)00404-?.

Fukuhara, N., Suzuki, M., Fujita, N., \& Tsubaki, T. (1975). Fabry's disease: on the mechanism of the peripheral nerve involvement. Acta Neuropathologica, 33, 9-21. doi:10.1007/BF00685960.

Gibas, A. L., Klatt, R., Johnson, J., Clarke, J. T., \& Katz, J. (2006). A survey of the pain experienced by males and females with Fabry disease. Pain Research \& Management, 11, 185-192.

Gijsbers van Wijk, C. M., van Vliet, K. P., Kolk, A. M., \& Everaerd, W. T. (1991). Symptom sensitivity and sex differences in physical morbidity: a review of health surveys in the United States and The Netherlands. Women \& Health, 17, 91-124. doi:10.1300/J013v17n01 06 .

Grabowski, G. A., \& Hopkin, R. J. (2003). Enzyme therapy for lysosomal storage disease: Principles, practice, and prospects. Annual Review of Genomics and Human Genetics, 4, 403-436. doi:10.1146/annurev.genom.4.070802.110415.

Hopkin, R. J., Bissler, J., \& Grabowski, G. A. (2003). Comparative evaluation of alpha-galactosidase-A infusions for treatment of Fabry disease. Genetics in Medicine, 5, 144-153. doi:10.1097/ 0L.GIM.0000069509.57929.CD.

Kahn, P. (1973). Anderson-Fabry disease: a histopathological study of three cases with observations on the mechanism of production of pain. Journal of Neurology, Neurosurgery, and Psychiatry, 366, $1053-1062$ 
Kralik, D., Brown, M., \& Koch, T. (2001). Women's experiences of 'being diagnosed' with a long-term illness. Journal of Advanced Nursing, 33, 594-602. doi:10.1046/j.1365-2648.2001.01704.x.

MacDermot, K. D., Holmes, A., \& Miners, A. H. (2001). AndersonFabry disease: Clinical manifestations and impact of disease in a cohort of 60 obligate carrier females. Journal of Medical Genetics, 38, 769-775. doi:10.1136/jimg.38.11.769.

MacDougall, V. L. (2002). Medical gender bias and managed care. Oklahoma City University Law Review. Oklahoma City University, 27, 781-910.

Malterud, K. (2000). Symptoms as a source of medical knowledge: understanding medically unexplained disorders in women. Family Medicine, 32, 603-611.

Malterud, K. (2001). The art and science of clinical knowledge: evidence beyond measures and numbers. Lancet, 358, 397-400. doi: $10.1016 /$ SO140-6736(01)05548-9.

Mehta, A., Ricci, R., Widmer, U., Dehout, F., Garcia de Lorenzo, A., Kampmann, C., et al. (2004). Fabry disease defined: baseline clinical manifestations of 366 patients in the Fabry Outcome Survey. European Journal of Clinical Investigation, 34, 236242. doi:10.1111/j.1365-2362.2004.01309.x.

Miles, M. B., \& Huberman, A. M. (1994). Qualitative data analysis: An expanded sourcebook. Thousand Oaks, California: Sage.

Morse, J. M. (1991). Is qualitative research an end in itself or the beginning of a process? In Qualitative Nursing Research: A Contemporary Dialogue. Newbury Park, California: Sage.

Pastores, G. M., \& Lien, Y. H. (2002). Biochemical and molecular genetic basis of Fabry disease. Journal of the American Society of Nephrology, 13, S130-S133. doi:10.1159/000048645.
Ries, M., Ramaswami, U., Parini, R., Lindblad, B., Whybra, C., Willers, I., et al. (2003). The early clinical phenotype of Fabry disease: a study on 35 European children and adolescents. European Journal of Pediatrics, 162, 767-772. doi:10.1007/ s00431-003-1299-3.

Ries, M., Wendrich, K., Whybra, C., Kampmann, C., Gal, A., \& Beck, M. (2001). Angiokeratoma and pain, but not Fabry's disease: considerations for differential diagnosis. Contributions to $\mathrm{Ne}$ phrology, 136, 256-259.

Ro, L. S., Chen, S. T., Tang, L. M., Hsu, W. C., Chang, H. S., \& Huang, C. C. (1999). Current perception threshold testing in Fabry's disease. Muscle \& Nerve, 22, 1531-1537 doi:10.1002/ (SICI) 1097-4598(199911)22:11<1531::AID-MUS7>3.0.CO:2-O.

Schiffmann, R., Kopp, J. B., Austin 3rd, H. A., Sabnis, S., Moore, D. F., Weibel, T., et al. (2001). Enzyme replacement therapy in Fabry disease: a randomized controlled trial. Journal of the American Medical Association, 285, 2743-2749. doi:10.1001 jama.285.21.2743.

Scott, L. J., Griffin, J. W., Luciano, C., Barton, N. W., Banerjee, T., Crawford, T., et al. (1999). Quantitative analysis of epidermal innervation in Fabry disease. Neurology, 52(6), 1249-1254.

Singh, B., Nunn, K., Martin, J., \& Yates, J. (1981). Abnormal treatment behaviour. The British Journal of Medical Psychology, 54, 67-73.

Thomas, J. (1993). Doing critical ethnography. Newbury Park, California: Sage.

Wang, R., Lelis, A., Mirocha, J., \& Wilcox, W. (2007). Heterozygous Fabry women are not just carriers, but have a significant burden of disease and quality of life. Genetics in Medicine, 9(1), 34-45. doi:10.1097/GIM.0b013e31802d8321. 